\title{
Microbiome: A New Lease to Microbiology
}

\author{
Vipin Gupta $^{1} \cdot$ Utkarsh Sood $^{2} \cdot \operatorname{Roshan~Kumar}^{3} \cdot$ Rup Lal $^{2} \cdot$ Vipin Chandra Kalia $^{4}$
}

Published online: 11 January 2020

(C) Association of Microbiologists of India 2020

The diversity of microbes and their pervasive influence on human beings is now well established. Microbiomes impact human beings, plants, animals and food products derived from them. The understanding of the interactions within microbiomes is advancing due to the rapid progress in sequencing technologies complemented by bioinformatics tools enabling generation of metadata in terms of metagenomics, proteomics, and metabolomics. The Human Microbiome Project (HMP) launched under the National Institutes of Health (NIH), USA program laid the foundation for generating in-depth information on microbes involved in oral health, birth mode, breast milk, infant gut, immunity and lifestyle disorders and its association with the host disease phenotype. Recent R\&D works have led to the development of microbiome-based innovative prevention and intervention strategies against a variety of diseases, establishment of biomarkers for disease risk and detection, cultivation of 'uncultivable' microbial diversity, and a robust understanding of factors affecting microbial community structure and function. The information from

Rup Lal

ruplal@gmail.com

Vipin Chandra Kalia

vckaliaku@gmail.com

1 Department of Zoology, University of Delhi, Delhi, India

2 The Energy and Resources Institute Darbari Seth Block, IHC Complex, Lodhi Road, New Delhi 110003, India

3 P.G. Department of Zoology, Magadh University, Bodh-Gaya, Bihar 824234, India

4 Department of Chemical Engineering, Konkuk University, 1 Hwayang-Dong, Gwangjin-Gu, Seoul 05029, Republic of Korea these studies has led to the development of translational research for new health interventions.

In contrast to global efforts on microbiome research, contributions from Indian R\&D organizations are rather scarce. In this special issue of Indian Journal of Microbiology, an update on current research on microbiomes in diverse areas has been compiled with a focus on chicken microbiome and its effect on human health, probiotics development as a remedy for microbiome dysbiosis, the interplay of the microbiome in health and wellness, its relation with hygiene and their role in environmental bioremediation. A detailed record of the taxonomically characterized and validated microbial species from India based on the works carried out during the last decade is a major highlight of this special issue of INJM. It supports the importance of microbial diversity present in Indian ecosystems. It also includes the original articles highlighting bacterial population dynamics in subgingival plaque and identification of biofilm inhibitors against two major human pathogens-Pseudomonas aeruginosa and Candida albicans. In addition, articles focusing on the microbial dynamics of food served in the University canteen in Portugal, marketed Ready-to-Eat Crickets and Gioddu provide critical insights into the presence of microbial communities in them, which certainly have a significant impact on the human health. Although, this special issue is a small step from the Indian continent, however, it is a hot spot for exploring microbiomes from diverse seasons, geographical regions, and ethnic populations. Efforts should be made to boost the microbiome research in India to harvest the fruits of the unique microbial diversity and use it for developing a sustainable economy.

Vipin Gupta (Guest Editor),

Utkarsh Sood (Guest Editor),

Roshan Kumar (Guest Editor),

Rup Lal (Editor),

Vipin Chandra Kalia (Editor in Chief) 